\title{
El aprendizaje basado en problemas en la enseñanza de hidrología
}

Zucarelli, Graciela Viviana* y Paris, Marta del Carmen*

\section{Resumen}

Este trabajo refiere a las estrategias de enseñanza-aprendizaje seleccionadas para la implementación en la Facultad de Ingeniería y Ciencias Hídricas (Universidad Nacional del Litoral, Santa Fe, Argentina) de una materia obligatoria de un programa de posgrado. En función de la temática del posgrado, el contenido y la ubicación de la materia dentro del plan de estudios, así como el perfil de los estudiantes, se estima conveniente utilizar estrategias didácticas que, a la vez de presentar conceptos teóricos básicos y específicos de Hidrología, conduzcan a desarrollar la visión global, trans e interdisciplinar de situaciones problemáticas. Se pretende además, favorecer el ejercicio de la participación, el intercambio de opiniones y consenso entre los profesionales especialistas.

En este caso, la técnica de Aprendizaje Basado en Problemas (ABP) se ha seleccionado como propuesta metodológica principal.

* Facultad de Bioquímica y Ciencias Biológicas. UNL.

e-mail: giraldez@fbcb.unl.edu.ar 